\title{
Monte Carlo Simulations of Signal Electrons Collection Efficiency and Development of New Detectors for ESEM.
}

\author{
V. Neděla ${ }^{1}$, I. Konvalina ${ }^{1}$, M. Oral ${ }^{1}$, J. Hudec ${ }^{1}$ \\ ${ }^{1}$ Environmental Electron Microscopy Group, Institute of Scientific Instruments of ASCR, \\ Královopolská 147, 61200 Brno, Czech Republic
}

This paper presents a study of the collection efficiency under various conditions, detector configurations and types of detected electrons in our environmental scanning electron microscope (ESEM) AQUASEM II. It is based on a Monte Carlo simulation of signal electron trajectories in vacuum and gaseous environment in a specially designed ionization detector of secondary electrons equipped with two circular electrodes, a drawing of which is in Fig. 1.

Four configurations of detection system were used for simulations. First, electrode A was connected to $+350 \mathrm{~V}$ and used to collect signal, while electrode B was grounded. Second, electrode A was grounded, while electrode B was connected to $+350 \mathrm{~V}$ and used to collect signal. Third, $+350 \mathrm{~V}$ was applied on electrodes $\mathrm{A}$ and $\mathrm{B}$ while signal was detected only from electrode A. Fourth, the same configuration as the third one, but signal was detected only from electrode B.

The simulation consisted of two steps. First, the spectrum of the signal electrons was computed by a Monte-Carlo method in the Geant4 [1]. Second, a dependence of the amplification coefficient on the energy and the pressure was obtained in the EOD (Electron Optical Design). The emission of the signal electrons from the gold surface was simulated using the Geant4 for 1000000 particles, the beam energy was $20 \mathrm{keV}$. The trajectories of signal electrons emitted from the sample to water vapor between the sample and the ionization detector and the signal amplification of the detected electrons were calculated using the EOD [2] software with a Monte Carlo plug-in [3]. 10000 signal electrons with the energies of 2.7, 4.7, 7.6, 50, 100, 500, 1000, 5000, 10000,15000 and $20000 \mathrm{eV}$ were traced through a $2 \mathrm{~mm}$ thick water vapor region (the sample-to-detection electrode distance) with the pressure of 50, 100, 200, .., $1000 \mathrm{~Pa}$.

The signals detected by the individual segments of the detector provide information about the final energy of the signal electrons. The distribution of the electrostatic fields between the detector and the sample, the spatial distribution of the signal electron emission from the sample as well as the gas type and the pressure all have a significant influence on the detected type of electrons.

Simulations of the electron collection efficiency for selected primary beam energies were run for a gold sample, taking into account the energy distribution of the signal electrons. The computed dependencies are in Fig. 2A. The results show, that electrode A collects both lowand high-energy electrons at the pressure of up $200 \mathrm{~Pa}$, while for the range of $200 \mathrm{~Pa}$ to $600 \mathrm{~Pa}$ the proportion of the high-energy electrons decreases significantly, almost vanishing above $600 \mathrm{~Pa}$. In the case of the second and fourth configuration, with the results in Figs. 2B and 2D, the low-energy electrons are significantly detected in the whole range of pressures; an increasing pressure causes the proportion of the high-energy electrons to increase, especially above $20 \mathrm{keV}$. In the third configuration, there is such an influence of the electric field of 
electrode A on the low-energy signal electrons, that their collection by electrode B is limited significantly. Predominantly high-energy electrons with energies of around $20 \mathrm{keV}$ are detected, as it is shown in Fig. 2C [4].
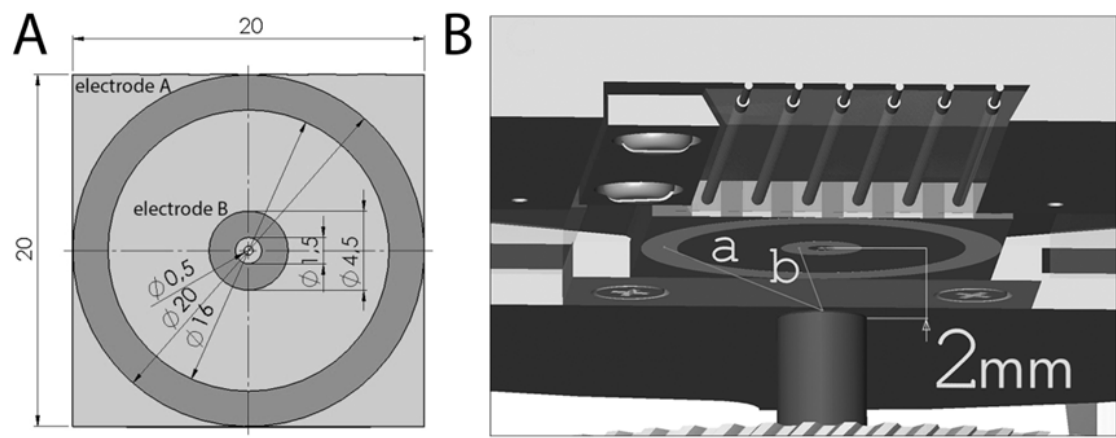

Figure 1. A) Detection electrodes of the ionization detector for ESEM, B) Experimental setup in ESEM AQUASEM II.
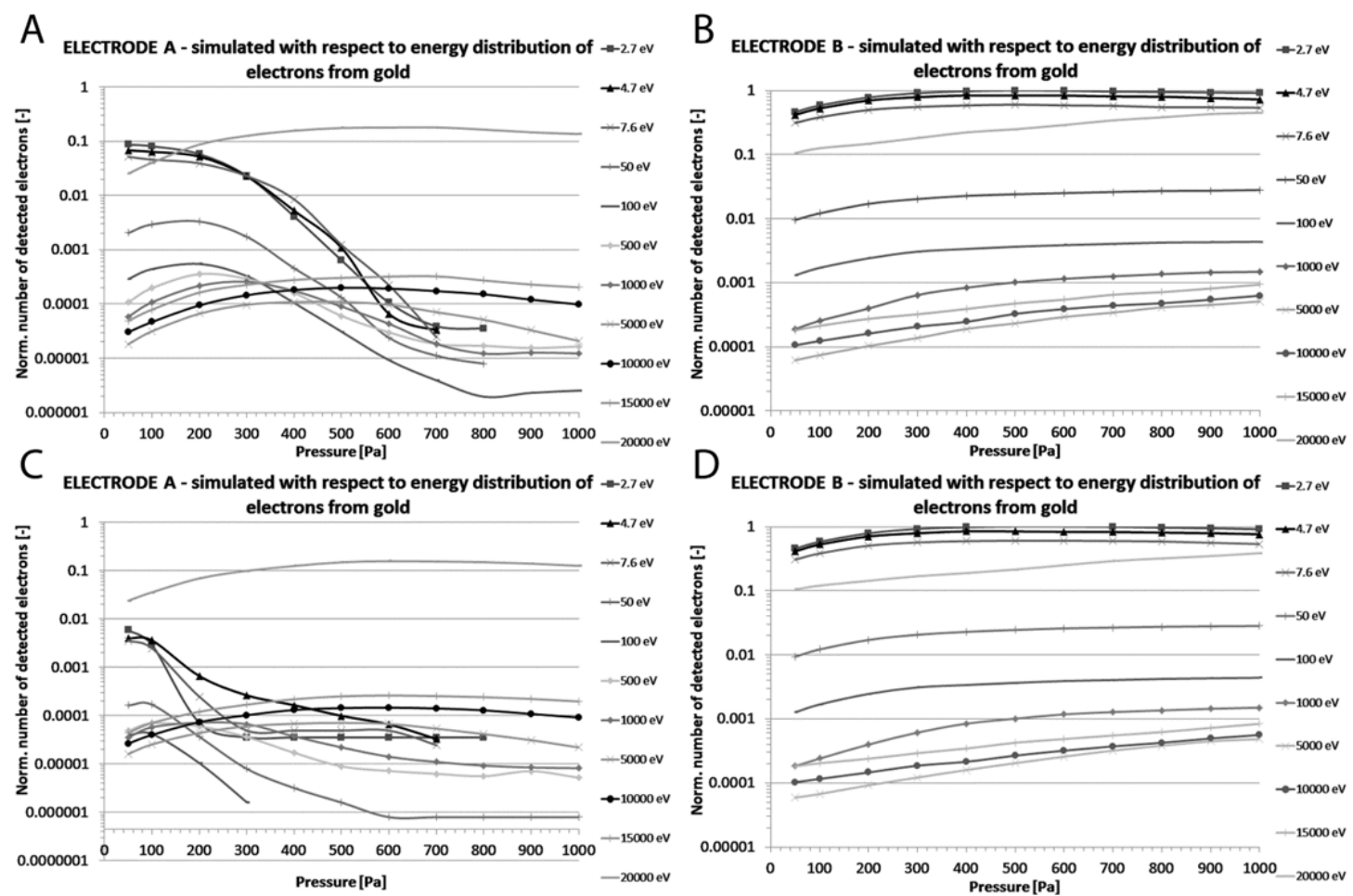

Figure 2. The dependence of the normalized number of electrons detected by electrodes A and B, see Fig.1, on the water vapor pressure and the energy of the signal electrons for four selected configurations (First-A, Second-B, Third-C, Fourth- D).

References:

[1] Agostinelli S et al., Nucl. Instr. and Meth. A 506 (2003), p. 250

[2] Lencová B, et al. Physics Procedia 1 (2008), p.315.

[3] Neděla V, et al., Nucl. Instrum. Methods in Physics A, 645 (1) (2011), p. 79.

[4] This work was supported by the grant No. GA 14-22777S and LO1212 together with the European Commission (ALISI No. CZ.1.05/2.1.00/01.0017). 